\title{
An Intelligent Assessment Method of Contact Fatigue Reliability for Rolling Bearing under EHL
}

\author{
Chunyu $\mathrm{Lu}^{\mathrm{a}, \mathrm{b}, *}$ and Shaojun $\mathrm{Liu}^{\mathrm{a}, \mathrm{b}}$ \\ ${ }^{a}$ School of Mechanical and Electrical Engineering, Central South University, Changsha 410083, CHINA \\ ${ }^{b}$ State Key Laboratory for High Performance Complex Manufacturing (Central South University), Changsha 410083, CHINA
}

\begin{abstract}
For the rolling bearing with expensive test cost or of inconvenient test, in order to efficiently and accurately analyze its contact fatigue reliability under elliptical contact elastohydrodynamic lubrication (EHL), an intelligent reliability assessment method is proposed. Contact stress under EHL is obtained by the mapping of oil film pressure, gotten by finite difference method (FDM), in the Hertz contact zone of the finite element model of rolling bearing. Considering the randomness of the EHL, material and fatigue strength correction factors, the limit state function is established by using artificial neural network (ANN). For finding the optimal reliability index and the design point, genetic algorithm (GA) based on normalized real number encoding is employed and the two adjusting factors are introduced into fitness function to resolve convergence and stability problem. Reliability sensitivity analysis is achieved by the advanced first-order secondmoment (AFOSM) method. Compared with the traditional Monte Carlo method (MCM), the proposed intelligent assessment method could embody the influence of EHL on contact fatigue reliability and has higher calculation efficiency and a wonderful global search capability in the whole optimization room.
\end{abstract}

Keywords: rolling bearing; artificial neural network (ANN); reliability; elastohydrodynamic lubrication (EHL); genetic algorithm (GA)

(Submitted on June 18, 2017; Revised on July 14, 2017; Accepted on August 2, 2017)

(O2017 Totem Publisher, Inc. All rights reserved.

\section{Introduction}

Reliability of rolling bearing directly affects the overall performance of various machineries and once rolling bearing failures, it will bring huge losses of life and property. Therefore, it is necessary to study reliability of rolling bearing. Currently, the main methods of evaluating contact fatigue reliability are statistical method, Monte Carlo method and response surface method (RSM) etc $[3,11,12,15,16,19]$. The statistical method is the most common method, but it requires a large number of fatigue data, which is not easy to be obtained. The Monte Carlo simulation method is implemented by using the computer, so test cost of the method is low very much, but its time cost is quite high. The RSM is an excellent reliability analysis method, which has all advantages (e.g. low test cost and short test time) of the above two methods. Except for the above contact fatigue reliability analysis methods, other methods are put forward by many experts and scholars at home and abroad $[8,10,14]$.

However, due to the function of lubricant in the actual working condition, a ball is separated from raceway by oil film thickness, which reduces wear and prolongs fatigue life that has exponential relation with the EHL film thickness [2,6,18,20]. Therefore, it is necessary to consider the influence of the EHL on contact fatigue reliability of rolling bearing. In order to integrate the effect of the EHL on contact fatigue reliability into reliability model, oil film pressure obtained by FDM is mapped into Hertz contact zone of finite element model of rolling bearing, thus the mapping relation between basic random variables and contact stress is established. Though the reliability analysis can be solved by using MCM, this method is too time-consuming, so it is difficult to accept. Generally, the Monte Carlo simulation method is used for assessing the effectiveness of other approximate reliability analysis methods. As for some rolling bearings with special structure or higher experimental cost, it is unscientific to use Monte Carlo method or the statistical method based on lots of bearing failure data. 
For the above-mentioned problem, according to ANN, normalized real-coded GA and AFOSM, an effective method is proposed for the contact fatigue reliability analysis of rolling bearing. The standard GA is easy to fall into local optimal solution and premature convergence. In order to overcome the above shortcomings, two adjusting factors are introduced in the fitness function. Considering the considerable differences between magnitudes of basic random variables, normalization operation is executed to guarantee the convergence of GA. Commonly, GA is only used to calculate maximum problem. However, for enabling GA to directly solve minimum problem, reciprocal of individual fitness is carried out in the Monte Carlo selection. Compared with the traditional MCM, the proposed method is more efficient and the relative error between the reliability of the two methods is only $0.75 \%$.

\section{Elliptical contact EHL and oil film pressure}

\subsection{Elliptical contact EHL model of rolling bearing}

Due to lubricant function, ball can not directly contact with raceway. Isothermal-ellipse-contact-elastohydrodynamic lubrications model has been introduced in detail in [18] and will be described briefly in this article.

- Relation between oil film pressure and other parameters is expressed in the Reynolds equation as follows:

$$
\frac{\partial}{\partial x}\left(\frac{\rho h^{3}}{\eta} \frac{\partial p}{\partial x}\right)+\frac{\partial}{\partial y}\left(\frac{\rho h^{3}}{\eta} \frac{\partial p}{\partial y}\right)=12 u \frac{\partial(\rho h)}{\partial x}
$$

Where $x$ and $y$ are the coordinate of two directions of orthogonal motion, respectively; $\rho$ and $\eta$ are the lubricant density and viscosity, respectively; $\mathrm{p}$ and $\mathrm{h}$ are the oil film pressure and thickness, respectively. $\mathrm{u}$ is the entrainment velocity between ball and raceway.

- Reynolds boundary conditions:

$$
\left\{\begin{array}{l}
p\left(x_{\text {in }}, y\right)=p\left(x_{\text {out }}, y\right)=p\left(x, y_{\text {in }}\right)=p\left(x, y_{\text {out }}\right)=0 \\
\partial p\left(x_{\text {out }}, y\right) / \partial x=0
\end{array}\right.
$$

Where $x_{\text {in }}$ and $x_{\text {out }}$ the entrance and the exit boundary of $x$ direction of the solution domain, respectively; $y_{\text {in }}$ and $y_{\text {out }}$ are the entrance and the exit boundary of $y$ direction of the solution domain, respectively;

- Elasticity equation

$$
h(x, y)=h_{0}+\frac{x^{2}}{2 R_{x}}+\frac{y^{2}}{2 R_{y}}+\frac{2}{\pi E} \iint_{\Omega} \frac{p(s, t)}{\sqrt{(x-s)^{2}+(y-t)^{2}}} d s d t
$$

Where $h_{0}$ is a constant; $R_{\mathrm{x}}$ and $R_{\mathrm{y}}$ the equivalent curvature radius between ball and raceway at the $x$ and $y$ direction, respectively; $E$ is the equivalent elasticity modulus; $s$ and $t$ are the additional coordinate corresponding to coordinate $x$ and $y$.

- $\quad$ Pressure-viscosity equation

Roelands equation that is closer to reality is adopted in pressure-viscosity relation.

$$
\eta=\eta_{0} \exp \left\{\left(I n \eta_{0}+9.67\right)\left[\left(1+p / p_{0}\right)^{z}-1\right]\right\}
$$

Where $\eta_{0}$ is the lubricant viscosity at ambient temperature; $p_{0}$ is the pressure coefficient; $z$ is the mineral oil coefficient, $z=0.68$.

- $\quad$ Pressure-density equation

For the accuracy of calculation, the following equation presented by Dowson, D. and Higginson [2] is applied in this study.

$$
\rho=\rho_{0}[1+0.6 p /(1+1.7 p)]
$$

Where $\rho_{0}$ is the lubricant density at ambient pressure. 
- $\quad$ Load equilibrium equation

$$
\int_{x_{\text {in }}}^{x_{\text {out }}} \int_{y_{\text {in }}}^{y_{\text {out }}} p(x, y) d x d y=\omega
$$

Where $\omega$ is the total load of Hertz contact zone. The above EHL equations are solved by using the FDM [7].

\subsection{Polynomial function fitting of oil film pressure}

Considering the complexity of solution process of EHL equations, Polynomial function is adopted to approximate the EHL equations to make the effect of EHL on contact stress to be integrated into limit state function.

In order to calculate conveniently and meet the accuracy requirement, oil film pressure is fitted by quadratic polynomial with intercrossing term to approximate the EHL equations, as follows.

$$
Y=a_{0}+\sum_{i=1}^{m} b_{i} X_{i}+\sum_{i=1}^{m} \sum_{j=1}^{m} c_{i j} X_{i} X_{j}
$$

Where $Y$ is oil film pressure; $X_{\mathrm{i}}(i=1,2, \ldots, \mathrm{m})$ is the random variables; $a_{0}, b_{\mathrm{i}}$, and $c_{\mathrm{ij}}$ are the unknown regression coefficient, $(\mathrm{m}+1) \cdot(\mathrm{m}+2) / 2$ in total.

Compared to the 2-level factor design, central composite design (CCD) has a higher fitting precision. Therefore, sample points are obtained by using CCD method.

\section{Intelligent analyses of reliability and sensitivity}

\subsection{Expression of reliability index}

According to the geometrical significance of reliability index, it is defined as the shortest distance between original point and limit state function in the standard normal space as follows [4,9]:

$$
\begin{aligned}
& \text { Minimize } \beta^{2}=x \cdot x^{T} \\
& \text { Subject to }: \mathrm{g}(x)=0
\end{aligned}
$$

Where $\beta$ is the reliability index of rolling bearing; $x=\left(x_{1}, x_{2}, \ldots, x_{\mathrm{n}}\right)$ is the basic random variable vector; $g(x)$ is the limit state function;

According to the EHL equations, the calculation of oil film pressure is complicated extremely, so the original limit state function $g(x)$ cannot directly established. In this study, explicit limit state function $g(x)$ is established by way of the combination between ANN and finite element method (FEM). For obtaining the minimum reliability index $\beta$, the GA based on real number encoding is employed.

\subsection{Artificial neural network based on CCD}

Artificial neural network is an information processing technique that is similar to the biological nerve system. It can adapt the external environmental change by means of study and is an intelligent method. Generally, ANN structure consists of input layer, hidden layer and output layer and the relation between layer and layer is established by way of weight value and threshold value. The neuron number of the input and the output layer depends on the number of input variables and output responses, respectively. As for the hidden layer, the optimal neuron number is obtained by trials. Since a 3-layer ANN model with a hidden layer has an enough ability to approximate nonlinear problem, the explicit limit state function of rolling bearing under EHL can be completed by using this ANN model. The mapping relation between hidden layer and input layer is logarithmic logistic transfer function "logsig" while the mapping relation between output layer and hidden layer is linear transfer function "purelin" [1,5].

Due to the huge difference between basic random variables, all the input and the output samples are normalized to prevent small number being ignored in the calculation process. In order to guarantee the calculation precision of ANN model, both train samples and test samples must come from the entire design space. Therefore, sampling method is important very much. 
CCD method is used to establish training datasets while Box-Behnken Matrix Sampling (BBM) method is used to establish test datasets. When the maximum number of epochs is reached or the target accuracy of the model is satisfied, the training process is terminated. The computation performance of the established ANN model is assessed by utilizing mean square error (MSE).

\subsection{Genetic algorithm based on normalized real number encoding}

After the explicit limit state function $g(x)$ is determined by the ANN method, the contact fatigue reliability of rolling bearing is finally obtained by the GA. In order to successfully achieve the proposed intelligent method in the study, many important improvements for GA are implemented including the introduction of two adjusting factors, the change of fitness function, and the combination between elitist strategy and Monte Carlo selection for selecting individual. Since the difference between random variables is greatly big, the normalized real number encoding method that makes every random variable to be transformed into the interval $[-1,1]$ is introduced to ensure the convergence of GA.

\subsubsection{Establishment of fitness function}

Generally, the GA is only used to handle the maximum problem by simulating heredity and evolution. In the study, for reducing the complexity of fitness function, the GA is adjusted by using special method depicted in section 3.3.3. Thus, the minimum problem of eq. (7) does not need to be transformed into the maximum problem. In order to keep more opportunities of reproduction for excellent individuals, restrict the amount of reproduction and make constrained problem of eq. (7) change into unconstrained problem, the exponential transformation of fitness function is performed and the adjusting factors $\kappa$ and $\gamma$ are introduced. To find the minimum reliability index, eq. (7) is replaced with the following fitness function, as follows:

$$
F(x)=\exp (\kappa \cdot(\beta+\gamma \cdot|g(x)|))
$$

Where $x=\left(x_{1}, x_{2}, \ldots x_{\mathrm{n}}\right)$ is the normalized random vector; $F(x)$ is the improved fitness function.

\subsubsection{Normalized real number encoding}

Binary-coded method is often used for traditional GA. However, this encoding method cannot search optimal value in entire real number space because of the limitation of binary number. In order to make search range extend to the entire variable space and avoid the phenomenon that huge number submerges small number, normalized real-coded method is used to solve the above problem.

\subsubsection{Monte Carlo selection}

Monte Carlo selection is one of the key steps of the GA. Its basic idea is that the probability of individual selected is inversely proportional to its fitness value.

Suppose that the population size is $N$, the fitness of individual $i$ is $T_{\mathrm{i}}$. For enabling GA to directly be used for the minimum problem, $T_{\mathrm{i}}$ is replaced with $1 / T_{\mathrm{i}}$ in Monte Carlo selection formula, as follows:

$$
P_{i}=\frac{1}{T_{i}} / \sum_{i=1}^{N} \frac{1}{T_{i}}
$$

Considering the randomness of genetic operation during the evolution of population, optimal individual of current population may be destroyed. In this study, Monte Carlo selection is combined with elitist strategy to solve this problem. The basic idea of Elitist strategy is that optimal individual of current population does not take part in crossover operation and mutation operation so that it can be used to replace the worst individual of the next generation population.

\subsection{Reliability sensitivity analysis}

According to the advanced first-order second-moment (AFOSM) theory, the first-order partial derivative of explicit limit state function $g(x)$ at the design point $x^{*}$ is the key of calculating reliability sensitivity.

According to the ANN theory, the mapping relations between layer and layer are shown as follows: 


$$
\begin{array}{r}
y_{j}=f_{1}\left(\sum_{i} \omega_{j i} x_{i}+\theta_{j}\right) \\
g_{k}=f_{2}\left(\sum_{j} \omega_{k j} y_{j}+\theta_{k}\right)
\end{array}
$$

Where $y_{\mathrm{i}}$ is the output of the $j$ th neuron of hidden layer; $g_{\mathrm{k}}$ is the output of the $k$ th neuron of output layer, namely, $g(x) ; f_{1}(\cdot)$ is the logarithmic transfer function 'sigmoid'; $f_{2}(\cdot)$ is the linear transfer function 'purelin'; $\omega_{\mathrm{ji}}$ is the connection weight between the $j$ th neuron of hidden layer and the $i$ th neuron of input layer; $\theta_{\mathrm{j}}$ is the threshold value of the $j$ th neuron of hidden layer; $\omega_{\mathrm{kj}}$ is the connection weight between the $j$ th neuron of hidden layer and the $k$ th neuron of output layer; $\theta_{\mathrm{k}}$ is the threshold value of the $k$ th neuron of output layer.

According to the derivation law of composite function, the first-order partial derivative of $g_{\mathrm{k}}$ with respect to $x_{\mathrm{i}}$ is calculated as follows:

$$
\frac{\partial g_{k}}{\partial x_{i}}=\frac{\partial g_{k}}{\partial y_{j}} \frac{\partial y_{j}}{\partial x_{i}}=\sum_{j}\left[\omega_{j i} \cdot \omega_{k j} \cdot f_{1}^{\prime}(\cdot) \cdot f_{2}^{\prime}(\cdot)\right]
$$

While reliability sensitivity is defined as the first-order partial derivative of the failure probability $P_{\mathrm{f}}$ with respect to distributed parameters of basic random variables, the reliability sensitivity can be calculated as follows:

$$
\begin{gathered}
\frac{\partial P_{f}}{\partial \mu_{x_{i}}}=-\left.\frac{\partial g_{k}}{\partial x_{i}}\right|_{x^{*}} \cdot \frac{\varphi(\beta)}{\sigma_{g_{k}}} \\
\frac{\partial P_{f}}{\partial \sigma_{x_{i}}}=\left.\frac{\partial g_{k}}{\partial x_{i}}\right|_{x^{*}} ^{2} \cdot \frac{\sigma_{x_{i}} \cdot \mu_{g_{k}} \cdot \varphi(\beta)}{\sigma_{g_{k}}^{3}}
\end{gathered}
$$

Where $\sigma_{\mathrm{gk}}$ and $\mu_{\mathrm{gk}}$ is the standard deviation and mean value of limit state function $g_{\mathrm{k}}$, respectively.

\section{Author Artwork}

For example, take a four-point contact ball bearing of a helicopter main reduction. Oil film pressure and contact stress are analyzed by the proposed method. The basic structure of four-point contact ball bearing is shown in Fig. 1.

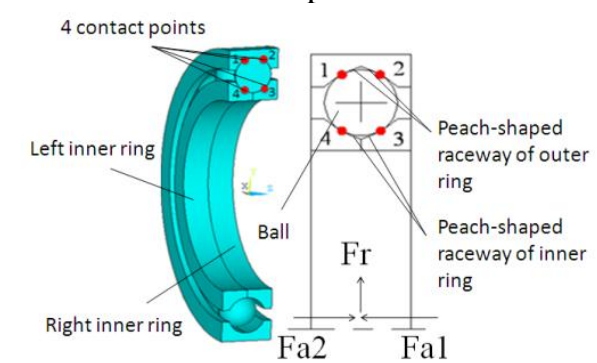

Fig.1 Radial sectioning of four-point contact ball bearing

In order to illustrate the superiority of the proposed method, MCM is also investigated. The computation time and the computation accuracy are used to assess the performance of the proposed method. Basic parameters and working conditions of four-point contact ball bearing are listed in Table 1.

\subsection{Analysis of mechanical model}

According to the simplification theory of two elastic objects, the contact between ball and raceway can be simplified as that between elastic ball and rigid plane, as shown in Fig. 2. $R_{\mathrm{x}}$ and $R_{\mathrm{y}}$ are the equivalent radiuses in $x z$ and $y z$ plane, respectively. $\omega$ is the normal load between ball and raceway. 
Table 1. Relevant parameters of four-point contact ball bearing

\begin{tabular}{|c|c|}
\hline Item & Parameter \\
\hline Inner diameter $/ \mathrm{mm}$ & 130 \\
\hline Outer diameter $/ \mathrm{mm}$ & 200 \\
\hline Width $/ \mathrm{mm}$ & 33 \\
\hline Ball diameter $/ \mathrm{mm}$ & 24 \\
\hline Number of ball $/ \mathrm{mm}$ & 17 \\
\hline Contact angle $\left./{ }^{\circ}\right)$ & 25 \\
\hline Gasket angle $/\left(^{\circ}\right)$ & 12 \\
\hline Rotational speed $/(\mathrm{r} / \mathrm{min})$ & 2400 \\
\hline Material & $\mathrm{GCr} 15 \mathrm{SiMnA}$ \\
\hline Elasticity $/ \mathrm{MPa}$ & $2.07 \times 10^{5}$ \\
\hline Poisson's ratio & 0.3 \\
\hline Fatigue limit/MPa & 1503.7 \\
\hline Kinematic viscosity $/\left(\mathrm{mm}^{2} / \mathrm{s}\right)$ & 4.11 \\
\hline &
\end{tabular}

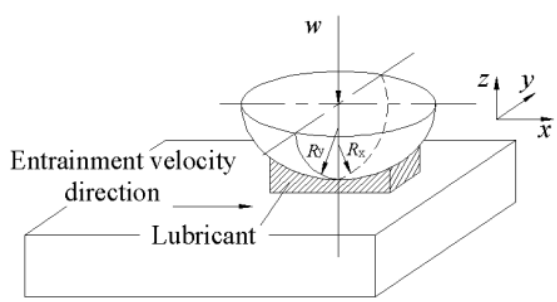

Fig.2 Simplified model of ball and raceway

Oil film pressure under EHL is obtained by the section 2.1, as shown in Fig. 3. There is a pressure peak near the exit of Hertz contact zone, which is consistent with actual cognition.

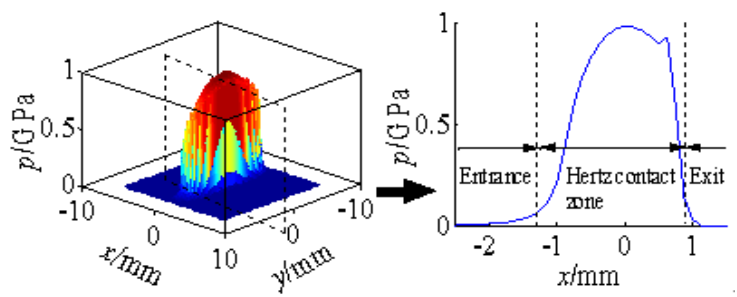

Fig.3 Distribution of oil film pressure in Hertz contact area

In order to embody the effect of oil film on contact stress of bearing in the limit state function, oil film pressure of the entire EHL solution zone is mapped into Hertz contact zone of finite element model of rolling bearing. The contact stresses are obtained by using the FEM [13]. For verifying the effectiveness of the mechanical model presented in this study, the contact stress of the center point of Hertz contact zone is calculated by using Hertz contact formula and the results are listed in Table 2.

Results show that the contact stresses under EHL are less than the Hertz contact stresses and their change tendencies are accordant. These results accord with the actual cognition. Therefore, the mechanical model presented in this study is effective for the calculation of contact stress of rolling bearing.

Table 2. Comparison of contact stress

\begin{tabular}{|c|c|c|}
\hline \multirow{2}{*}{ Axial load/N } & \multicolumn{2}{|c|}{ Contact stress/MPa } \\
\cline { 2 - 3 } & Proposed model & Hertz formula \\
\hline 2300 & 653.99 & 811.59 \\
\hline 2400 & 656.26 & 821.01 \\
\hline 2500 & 658.37 & 830.22 \\
\hline 2600 & 666.98 & 839.22 \\
\hline 2700 & 667.99 & 848.02 \\
\hline
\end{tabular}




\subsection{Polynomial fitting of oil film pressure}

\subsubsection{Basic random variables}

According to [17], the distribution types, mean value and standard deviation of random variables required for this problem are determined as shown in Table 3. In addition, random variable $v_{0}$ and $N$ is determined based on the actual measure values. In spite of the correlation between several basic random variables, all the basic random variables are assumed to be statistically independent in this study.

Table 3. Stochastic parameters and distribution types

\begin{tabular}{|c|c|c|c|}
\hline Item & PDF & Mean & COV \\
\hline Rotational speed, $N /(\mathrm{r} / \mathrm{min})$ & DAUS & 2355 & 0.02 \\
\hline Material density, $\rho /\left(\mathrm{kg} / \mathrm{m}^{3}\right)$ & DAUS & 7800 & 0.05 \\
\hline Elasticity modulus, $E / \mathrm{Pa}$ & DAUS & $2.07 \mathrm{E}+011$ & 0.03 \\
\hline Poisson's ratio, $\lambda$ & DAUS & 0.30 & 0.01 \\
\hline Initial viscosity, $v_{0} / \mathrm{Pa} \cdot \mathrm{s}$ & DAUS & 0.017 & 0.01 \\
\hline Surface quality coefficient, $\beta$ & DAUS & 1 & 0.01 \\
\hline Effective stress concentration coefficient, $K_{\sigma}$ & DAUS & 1.75 & 0.02 \\
\hline Size coefficient, $\varepsilon$ & DAUS & 0.92 & 0.06 \\
\hline Material contact fatigue limit, $\sigma_{-1} / \mathrm{MPa}$ & DAUS & 1503.7 & 0.03 \\
\hline
\end{tabular}

\subsubsection{Basic random variables}

According to the solution process of elliptical contact EHL model, basic random variables $N, E, \quad \lambda$ and $v_{0}$ have influence on oil film pressure. Therefore, they are used to fit oil film pressure. Suppose the maximum oil film pressure of Hertz contact zone between maximum loaded rolling element and inner raceway is $p_{s}$, according to section 2.2 , polynomial function of $p_{s}$ is finally fitted by eq. (6), as shown in eq. (15).

$$
\begin{aligned}
p_{s}= & -2.9016 \times 10^{10}+8.371 \times 10^{6} N+2.1893 \times 10^{12} v_{h}- \\
& 5.663 \times 10^{-6} N \cdot E-2.0643 \times 10^{8} N \cdot v_{h}+1.546 E \\
& v_{h}-7.7889 \times 10^{2} N^{2}--5.9849 \times 10^{13} v_{h}^{2}
\end{aligned}
$$

\subsection{Establishment of limit state function}

According to the stress-strength interference theory, the limit state function of rolling bearing is defined as follows:

$$
Y=\sigma_{p}-S_{\max }
$$

Where $S_{\max }$ is the maximum contact stress obtained by way of section $4.1 ; \sigma_{\mathrm{p}}$ is the contact fatigue strength limit, as follows:

$$
\sigma_{p}=\varepsilon \cdot \beta \cdot \sigma_{-1} / K_{\sigma}
$$

\subsection{Estimation of reliability index and design point by means of GA-based ANN}

Table 4 shows the relevant parameters of ANN and GA. After the ANN is trained, the MSE of the training datasets is $2.187 \times 10^{-5}$, meeting the error performance target. ANN is tested as shown in Fig. 4. Obviously, predicted values are close to original values, which indicates that the approximated limit state function $g(x)_{\mathrm{ANN}}$ can replace the real limit state function $g(x)$. 
Table 4. Design parameters of ANN and GA

\begin{tabular}{|c|c|c|}
\hline Item & Parameter name & value \\
\hline \multirow{4}{*}{ ANN } & Neuron number of input layer & 9 \\
\cline { 2 - 3 } & Neuron number of output layer & 1 \\
\cline { 2 - 3 } & Neuron number of hidden layer & 9 \\
\cline { 2 - 3 } & Number of training datasets & 147 \\
\cline { 2 - 3 } & Number of testing datasets & 121 \\
\hline \multirow{4}{*}{ GA } & Population size & 80 \\
\cline { 2 - 3 } & Crossover probability & 0.9 \\
\cline { 2 - 3 } & Mutation probability & 0.03 \\
\cline { 2 - 3 } & Maximum number of iterations & 500 \\
\cline { 2 - 3 } & Adjusting factor of penalty function & \multirow{2}{*}{$\kappa=5 \times 10^{-4}$} \\
\cline { 2 - 3 } & & $\gamma=10^{4}$ \\
\hline
\end{tabular}

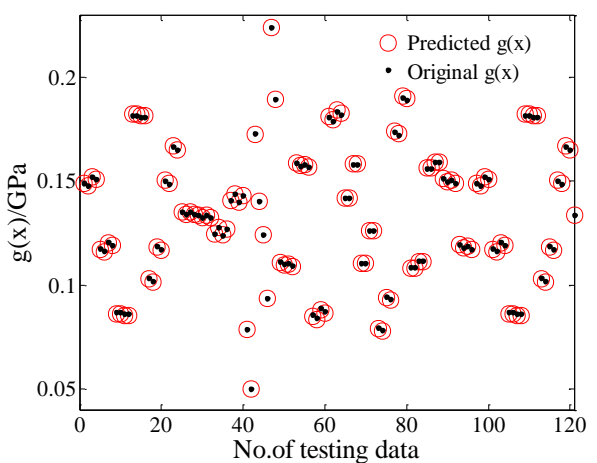

Fig.4 Test of artificial neural network

The evolution of population and optimal chromosome is shown in Fig. 5. Optimal design point and fitness value are listed in Table 5. The optimized process of the reliability index $\beta$ is shown in Fig. 6 . The minimum reliability index is 2.609 and appears at the 480th generation. All simulation results are achieved on Shuguang 5000 high performance computing platform.

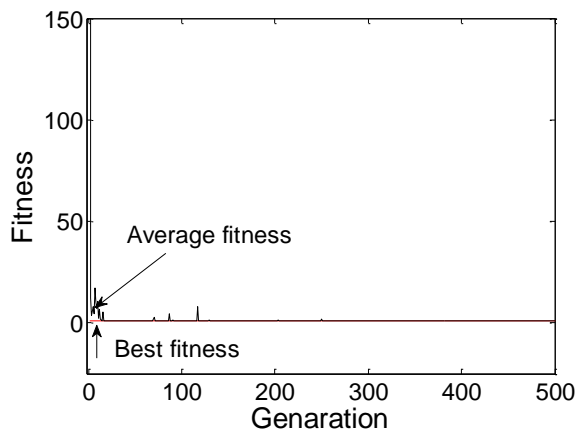

Fig.5 Fitness change of genetic algorithm

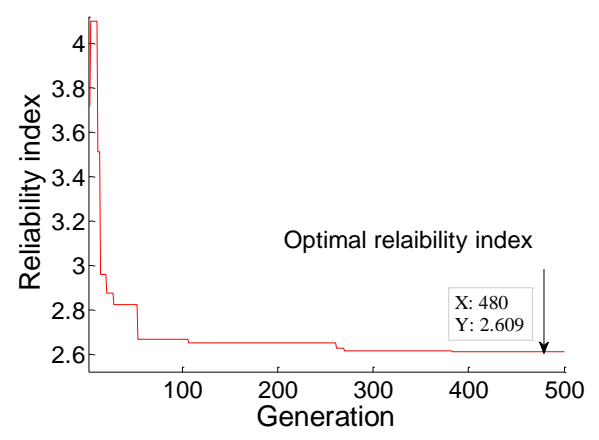

Fig.6 Optimization curve of reliability index

Table 5. Optimal design point and fitness

\begin{tabular}{|c|c|c|}
\hline Item & symbol & value \\
\hline Fitness & & 1.0013 \\
\hline \multirow{4}{*}{ Design point } & $N^{*}$ & $2.3401 \times 10^{3}$ \\
\cline { 2 - 3 } & $\rho^{*}$ & $7.5135 \times 10^{3}$ \\
\cline { 2 - 3 } & $E^{*}$ & $2.0422 \times 10^{11}$ \\
\cline { 2 - 3 } & $\lambda^{*}$ & $3.0047 \times 10^{-1}$ \\
\cline { 2 - 3 } & $v_{0}^{*}$ & $1.7078 \times 10^{-2}$ \\
\cline { 2 - 3 } & $\beta^{*}$ & $9.8982 \times 10^{-1}$ \\
\cline { 2 - 3 } & $K_{\sigma}^{*}$ & $1.7703 \times 10^{0}$ \\
\cline { 2 - 3 } & $\varepsilon^{*}$ & $9.8224 \times 10^{-1}$ \\
\cline { 2 - 3 } & $\sigma_{-1}^{*}$ & $1.4395 \times 10^{3}$ \\
\hline
\end{tabular}




\subsection{Assessment of reliability sensitivity}

Reliability sensitivity results are shown in Eq.18 and Eq.19, which are obtained by section 3.4. The normalized sensitivity vector $\partial \mathrm{P}_{\mathrm{f}} / \partial \mathrm{u}_{\mathrm{X}}$ of failure probability on mean values of basic random variables shows that the reliability increases as the mean values of $N, v_{0}, \beta, \varepsilon, \sigma_{-1}$ and $\rho$, but the mean values of $E, \lambda, k_{\sigma}$ have an inverse influence. Those conclusions are consistent with the actual cognitions. According to [18], the increments of $N$ and $v_{0}$ are good for improving the contact conditions of the rolling bearing and prolong the contact fatigue life; The increments of $E$ and $\lambda$ make equivalent elastic modulus $E^{\prime}$ higher, which will lead to contact zone between ball and raceway smaller, thus worsen the contact conditions. The effects of $\beta, \varepsilon, k_{\sigma}$ and $\sigma_{-1}$ can be deduced based on the Eq. (17).

According to the normalized sensitivity vector $\partial \mathrm{P}_{\mathrm{f}} / \partial \sigma_{\mathrm{X}}$, the reliability of rolling bearing under EHL decreases as the standard deviation of the basic random variables increase.

$$
\begin{aligned}
& \frac{\partial \boldsymbol{P}_{\mathrm{f}}}{\partial \boldsymbol{\mu}_{\mathrm{X}}}=\left[\begin{array}{l}
\partial P_{\mathrm{f}} / \partial \mu_{\mathrm{N}} \\
\partial P_{\mathrm{f}} / \partial \mu_{\mathrm{\rho}} \\
\partial P_{\mathrm{f}} / \partial \mu_{\mathrm{E}} \\
\partial P_{\mathrm{f}} / \partial \mu_{\lambda} \\
\partial P_{\mathrm{f}} / \partial \mu_{v_{0}} \\
\partial P_{\mathrm{f}} / \partial \mu_{\beta} \\
\partial P_{\mathrm{f}} / \partial \mu_{\mathrm{K}_{\sigma}} \\
\partial P_{\mathrm{f}} / \partial \mu_{\varepsilon} \\
\partial P_{\mathrm{f}} / \partial \mu_{\sigma_{-1}}
\end{array}\right]=\left[\begin{array}{c}
-8.3468 \times 10^{-3} \\
-8.9345 \times 10^{-3} \\
2.0454 \times 10^{-2} \\
1.0037 \times 10^{-3} \\
-4.4145 \times 10^{-3} \\
-1.0824 \times 10^{-1} \\
1.1337 \times 10^{-1} \\
-4.7765 \times 10^{-1} \\
-8.5695 \times 10^{-1}
\end{array}\right] \\
& \frac{\partial \boldsymbol{P}_{\mathrm{f}}}{\partial \boldsymbol{\sigma}_{\mathrm{X}}}=\left[\begin{array}{l}
\partial P_{\mathrm{f}} / \partial \sigma_{\mathrm{N}} \\
\partial P_{\mathrm{f}} / \partial \sigma_{\rho} \\
\partial P_{\mathrm{f}} / \partial \sigma_{\mathrm{E}} \\
\partial P_{\mathrm{f}} / \partial \sigma_{\lambda} \\
\partial P_{\mathrm{f}} / \partial \sigma_{v_{0}} \\
\partial P_{\mathrm{f}} / \partial \sigma_{\beta} \\
\partial P_{\mathrm{f}} / \partial \sigma_{\mathrm{K}_{\sigma}} \\
\partial P_{\mathrm{f}} / \partial \sigma_{\varepsilon} \\
\partial P_{\mathrm{f}} / \partial \sigma_{\sigma_{-1}}
\end{array}\right]=\left[\begin{array}{l}
9.3473 \times 10^{-5} \\
1.0710 \times 10^{-4} \\
5.6128 \times 10^{-4} \\
1.3516 \times 10^{-6} \\
2.6146 \times 10^{-5} \\
1.5718 \times 10^{-2} \\
1.7243 \times 10^{-2} \\
3.0609 \times 10^{-1} \\
9.8527 \times 10^{-1}
\end{array}\right]
\end{aligned}
$$

\section{Method discussion}

The results of the proposed method are compared with those obtained by traditional MCM as listed in $=$ Table 6 . Obviously, the proposed method is accurate and efficient according to the reliability index $\beta$, number of test and time consumption. The success of the proposed method mainly depends on whether the two adjusting factors $\gamma$ and $\kappa$ are able to get the proper values. The following will illustrate the influence of the adjusting factors on the stability.

Considering the randomness of the initial population, the reliability index may be different for each calculation, so the reliability analysis is executed 10 times by the proposed method for each adjusting factor. All the analysis results are used for the assessment of the stability of the proposed method. Figure 7 shows that the reliability index fluctuates greatly and is far away from the exact solution without the adjusted factor $\kappa$. When the $\kappa$ increases from $10^{-5}$ to $10^{-3}$, the reliability index becomes more and more stable and close to the exact solution. In comparison with conclusions of the $\kappa$, Figure 8 indicates that the adjusting factor $\gamma$ has the similar rule. Through the above analysis, the optimal adjusting factors are determined.

Table. 6 Comparison of the proposed method and tradition MCM

\begin{tabular}{ccccccc}
\hline Method & $\beta$ & $R$ & $R_{\mathrm{e}} / \%$ & Number of test & Computing speed of Shuguang platform/TFlops & Time \\
& & & 147 & 10 & 0.74 \\
ANN-GA & 2.609 & 0.99546 & 0.75 & 200000 & 617 \\
\hline
\end{tabular}




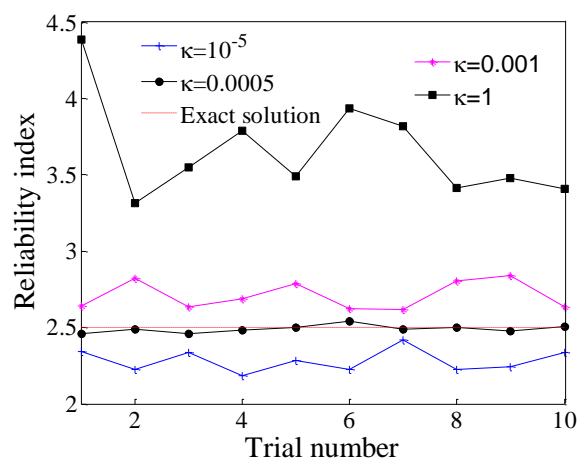

Fig.7 Influence of adjusting factor $\kappa$

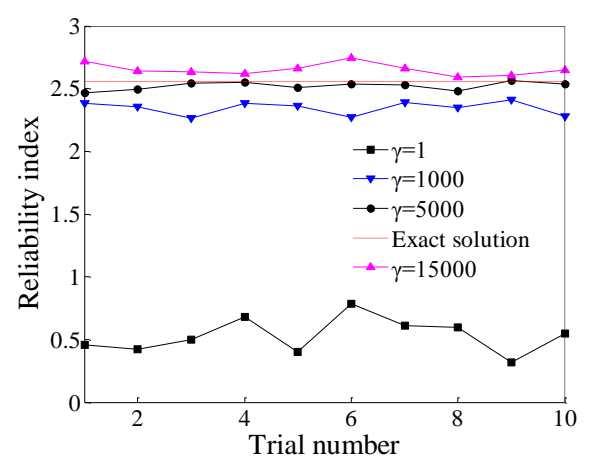

Fig.8 Influence of adjusting factor $\gamma$

\section{Conclusions}

1) The influence of EHL on contact fatigue reliability is integrated into the Polynomial response surface function successfully by way of mapping of oil film pressure in the hertz contact zone. The reliability sensitivity results obtained by the proposed method in this study are in accordance with the actual cognition, which indicates that the contact fatigue reliability analysis model proposed in this paper about four-point contact ball bearing is effective.

2) The method that the selection probability is obtained by the reciprocal fitness effectively avoids the transformation from maximum problem to minimum problem and simplifies the fitness function. Thus, GA can be directly applied to minimum problem.

3) The normalization of basic random variables successfully overcomes the difficulty that huge number submerges small number during the calculation.

4) In comparison with the traditional MCM, the superiority of the proposed method (GA-based ANN) is embodied not only in the efficiency but also in the accuracy, which can be observed through the reliability results and the consuming time.

5) The introduction of normalized real number encoding and two adjusting factors simplifies the contact fatigue reliability analysis model and enhances the stability of GA.

\section{Acknowledgements}

This research is supported by High Performance Computing Center of CSU of China, Hunan Provincial Innovation for Postgraduate of China (CX2014B060) and General Armament Pre-research Foundation of China (813XXXX).

\section{References}

1. S. Chatterjee and S. Bandopadhysy, "Reliability Estimation Using A Genetic Algorithm-based Artificial Neural Network: An Application to A Load-haul-dump Machine", Expert Systems with Applications, vol. 39, no. 12, pp. 10943-10951,2012.

2. P. H. Dawson, "Effect of Metallic Contact on The Pitting of Lubricated Rolling Surface", Journal of Mechanical Engineering Science, vol. 7, no. 1, pp. 147-155, 1962.

3. X. H. Gao, X. D. Huang and R. J. Hong, "A Rolling Contact Fatigue Reliability Evaluation Method and Its Application to A Slewing Bearing", Journal of Tribology, vol. 134, no. 1, pp. 1-7, 2012.

4. J. S. Gui, H. Liu and H. G. Kang, "An Intelligent Method for Structure Reliability Analysis Based on Response Surface”, China Ocean Engineering, vol. 18, no. 4, pp. 653-661, 2004.

5. B. Hariprasad, P. Bhattacharjee and A. Venugopal, "Prediction of Vehicle Reliability Using ANN", International Journal of Performability Engineering, vol. 8, no. 3, pp. 321-329, 2012.

6. Y. Hu, S. J. Liu and S. Ding, "Application of Response Surface Method for Contact Fatigue Reliability Analysis of Spur Gear with Consideration of EHL", Journal of Central South University, vol. 22, no. 7, pp. 2549-2556, 2015.

7. P. Huang, "Numerical Calculation of Elastohydrodynamic Lubrication: Methods and Programs", Tsinghua University Press, 2015.

8. D. Y. Jiang, T.Y. Wang and Y.X. Jiang, "Reliability Analysis of Motor Spindle Bearing Based on Operating Condition", IEEE Computer Society, vol. 2, pp. 989-992, 2011.

9. C. Jin, "An Artificial Neural Network Based Genetic Algorithm for Estimating the Reliability of Long Span Suspension Bridges", Finite Element in Analysis and Design, vol. 46, no. 8, pp. 658-667, 2010.

10. H. K. Li, Z. X. Zhang and X. G. Li, "Reliability Estimation Based on Moving Average and State Space Model for Rolling Element Bearing", Journal of Shanghai Jiao tong University (Science), vol. 20, no. 3, pp. 317-321,2015. 
11. J. Liu and Y. Li. “An Improved Adaptive Response Surface Method for Structural Reliability Analysis”, Journal of Central South University, vol. 19, no. 4, pp. 1148-1154,2012.

12. J. Malinowski, "A Monte Carlo Method for Estimating Reliability Parameters of A Complex Repairable Technical System with Inter-component Dependencies", IEEE Transactions on reliability, vol. 62, no. 1, pp.256 -266,2013.

13. V. Monfared, "Contact Stress Analysis in Rolling Bodies by Finite Element Method (FEM) Statically", Journal of Mechical Engineering and Automation, vol. 2, no. 2, pp. 12-16,2012.

14. T. Most and T. Knabe, "Reliability Analysis of Bearing Failure Problem Considering Uncertain Stochastic Parameters", Computers and Geotechnics, vol. 37, no. 3, pp. 299-310,2010.

15. R. Sehgal, O. P. Gandhi and S. Angra, "Reliability Evaluation and Selection of Rolling Element Bearings", Engineering and System Safety, vol. 68, pp. 39-52, 2000.

16. A. A. Shinde, R. G. Desavale and S.G. Kumbbar, "Theoretical and Experimental Studies on Vibrations Produced by Defects in Doubble Row Ball Bearing Using Response Surface Method”, International Journal of Research in Engineering and Technology, vol. 3, no. 7, pp. 140-145, 2014.

17. Z. L. Sun and L. Y. Chen, "Theory and Method of Utility Mechanical Reliability Design", Science Press, 2003.

18. S. Z. Wen, P. R. Yang, "Elastohydrodynamic Lubrication", Tsinghua University Press, 1992.

19. S. M. Zaharia and C. O. Morariu, "Simulation and Analysis of the Milling Machines Reliability Using the Monte Carlo Method", Research \&Science Today, vol.2, no. 10, pp. 108-114,2015.

20. E. V. Zaretsky, L. B. Sibley and W.J. Anderson, "The Role of Elastohydrodynamic Lubrication in Rolling Contact Fatigue", Journal of Fluids Engineering, vol. 85, no. 3, pp. 439-477,1963.

Chunyu Lu will receive doctor degree in Mechanical Electronics Engineering from Central South University, Changsha, China, in 2017. His current research interests include structure fatigue, product reliability and rolling bearing analysis.

Shaojun Liu, Ph.D., is a professor in Mechanical Electronics Engineering, Central South University. His current research interests include deep-sea equipment and hybrid power technology. 